\title{
The Physician-Patient Relationship in Dementia Care
}

\author{
David M. Blass \\ Abarbanel Mental Health Center, Bat Yam, Israel, affiliated with the Sackler School of Medicine, Tel Aviv University, Tel Aviv, \\ Israel; and the Department of Psychiatry and Behavioral Sciences and the Berman Institute of Bioethics, Johns Hopkins Medical \\ Institutions, Baltimore, Maryland
}

\begin{abstract}
Summary: Medical care of patients with dementia often occurs within a physician-patient relationship whose features differ from relationships with patients without dementia. Many basic assumptions of the physician-patient relationship may not completely hold true, and certain aspects of the patient role may be shared by others besides the patient. For example, the entire premise of consent to the patient role may be inapplicable to patients who lack insight into their illness. In addition, caring for cognitively impaired patients who do not comprehend the purpose of the physician-patient interaction may render physicians vulnerable to losing empathy with their patients and
\end{abstract}

objectifying them. This can lead to viewing patients as collections of symptoms rather than as humans suffering with illnesses and burdens. The fact that certain medical interventions, such as treatment of neuropsychiatric disturbances that do not trouble the patient, may appear to be initiated for the primary purpose of alleviating caregiver emotional stress also affects the physician-patient relationship. The present review examines how this relationship may be altered and presents a framework within which these alterations can be considered. Key Words: Physician-patient relationship, dementia care, paternalism, diagnostic disclosure.

\section{INTRODUCTION}

Several universal moral principles, such as respect for the sanctity of human life, hold true within the physician-patient relationship under all circumstances. Nonetheless, the application of certain other values may be complicated by features related to the context within which the relationship exists, or by patient characteristics. ${ }^{1}$ For example, a military physician must treat a soldier, thus facilitating the soldier's return to battle even though from a health perspective this may not be in the soldier's best interest. In this case, the context of the relationship (i.e., the military) in part determines what are considered valid goals of care.

Similarly, medical care for patients with dementia poses certain challenges in the application of ethical principles to clinical decisions, or even to the entire framework of clinical care. Many of the basic assumptions of the physician-patient relationship may not completely hold true, and certain aspects of the patient role may be shared by others besides the patient. ${ }^{2}$ Because many patients with dementia lack insight into their ill-

Address correspondence and reprint requests to: David M. Blass, MD, Abarbanel Mental Health Center, 15 Keren Kayemet L'Yisrael Street, Bat Yam, 59436 Israel. E-mail: dmblass@jhmi.edu. ness, the entire premise of consent to the patient role may have lost its foundation. Treatments may be initiated to combat the patient's symptoms with the goal also of reducing caregiver burden. These examples, among many others familiar to the clinician, raise ethical challenges within the physician-patient relationship, challenges that require exploration. In this article a number of these issues are reviewed, and approaches to resolving some of these problems are suggested.

\section{HOW DOES DEMENTIA AFFECT THE ESTABLISHMENT OF THE PHYSICIAN-PATIENT RELATIONSHIP?}

In the traditional Western model of the physicianpatient relationship, the patient is an individual, a person who may or may not allow information or influence to be shared with others. In circumstances such as a healthy marital relationship or a cognitively intact elderly parent living with adult children, information and influence may be shared as a matter of course. This sharing, when it occurs, results from consent (ideally, explicit consent) by the patient to thus construct the treatment setting, and it need not be automatic. Clinical care is often enhanced by the involvement of a number of parties, and clinicians may encourage this arrangement, but it is not mandatory. 
In the case of a patient with dementia, the situation is more complicated, in ways that might vary based on the severity of the illness. Full participation in any medical evaluation is generally predicated upon an understanding of and consent to the evaluation process itself, a mutual agreement as to the reason for the evaluation and the nature of the clinical problem to be addressed, as well as an understanding of and ability to consent to the recommended treatment. These aspects of participation may, to a greater or lesser degree, be lacking in the initial dementia assessment. ${ }^{3}$

How does the lack of patient participation affect the establishment of the physician-patient relationship? The patient may have initially been brought to the clinic under false or misleading pretenses. The evaluation may have been explained to the patient as being for 'sleep problems' or for some other problem only marginally related to the actual focus of the evaluation. Although from the family's perspective this may have been justifiable as the only way to get the patient the necessary help, the physician who does not yet have an established relationship with the patient is placed in the position of having to evaluate a somewhat unwilling patient. This situation is similar to that generally faced by psychiatrists when evaluating patients who lack insight into their symptoms. In that case however, the possibility always exists that with treatment the patient's insight will improve, allowing the physician-patient relationship to achieve its usual features. In the case of the patient with dementia, it is known from the beginning of treatment that this insight will likely never develop.

There is no simple solution to this conflict for the physician, particularly for a new patient about whom the physician has little knowledge and with whom no preexisting relationship exists. There is also no way to get the needed information to clarify the situation other than to proceed with the evaluation itself. On the one hand, for a physician who treats dementia patients to refuse under all circumstances to evaluate patients who say they do not want to be evaluated is simply not humane, for both patients and families.

This assessment emerges from a combination of factors. The first is that of all patients referred for cognitive assessment, a significant percentage do in fact have a cognitive impairment. Second, due to that cognitive impairment, many patients have inadequate insight into either the need for evaluation or the evaluation process itself. Third, a dementia assessment per se is a low-risk, low-discomfort procedure and may ultimately provide critical information for the patient's well-being and future care. Finally, clinical experience suggests that in most cases of patients who initially refuse a dementia evaluation the objection is not sustained and dissipates once the patient becomes familiar with the physician, staff, and setting. Thus, the physician has to balance the significant possibility of being presented with a patient who has impaired capacity to consent to or to refuse medical assessments or interventions with an opposing possibility, that the patient's refusal in fact reflects the will of an individual with intact decisional capacity.

When the patient does not object to the evaluation itself but simply has little or no insight into the reason for the assessment, the situation is less of an ethical dilemma than a deviation from the usual physician-patient relationship, in that no mutually shared agenda exists. This lack of agenda can be problematic for the physicianpatient relationship on a practical as well as an emotional level. Practically, this lack of insight complicates the implementation of clinical recommendations, particularly when they entail restriction of activities that impose a measure of hardship upon the patient, such as cessation of driving or cooking. The assistance of family members must often be enlisted to devise a plan for implementation and enforcement of the restrictions.

Emotionally, this lack of sharing an agenda creates challenges for the physician that are an extension of the emotional challenges experienced by the family of a dementia patient, but that also relate to the unique psychological challenges faced by physicians caring for dementia patients. Among the challenges dementia caregivers face is the difficulty of preserving the internal representation of the individual as he or she was while contending with the sense that in important ways the patient has somehow become 'another.' This aspect of dementia care is very painful for families, heightening caregiver burden and generating feelings of loss. For physicians likewise, when a patient lacks rudimentary insight into the purpose of the encounter it contributes to the sense that the individual has been lost, or greatly diminished.

Additionally, for the physician caring for dementia patients, lack of insight on the part of the patient presents a unique psychological challenge. In any field of medicine, repeated exposure to suffering, disability, and death renders physicians vulnerable to losing empathy with their patients and objectifying them, viewing them as collections of symptoms rather than as humans suffering with illnesses and burdens. In dementia care, a number of factors magnify this challenge for physicians. Loss of cognitive function generally, and the lower level of participation in the medical interview and decision-making specifically, emphasize the transformation and loss of standing in the adult sphere that the patient has undergone.

With patients whose verbal abilities are greatly impaired, or whose care is discussed with families without their presence, the risk of objectification increases. This risk is greater still when plans for treatment and safety and even a change of residence are developed without significant input from the patient. When the patient is 
unable to even comprehend the purpose of the medical encounter, it serves at the outset of the physician-patient relationship to make the physician vulnerable to the psychological dynamic of objectification. This aspect of dementia care, relating to the professionalism and humanity of the physician, is an important but easily neglected requisite component of the ongoing emotional effort involved in caring for patients with dementia.

\section{DISCLOSURE OF DEMENTIA DIAGNOSIS}

There is conflicting evidence regarding what caregivers, professionals, and patients themselves think patients should be told about their diagnosis of dementia. ${ }^{4} \mathrm{~A}$ number of studies indicate that individuals without dementia report wanting to be told of their diagnosis should they ever develop dementia, but that they have reservations about sharing similar information with a family member should that family member ever be diagnosed with dementia. ${ }^{5,6}$ Studies of clinical practice reveal differing approaches among clinicians to the issue of sharing the diagnosis. ${ }^{7}$

Although many clinicians feel that the ideal is to share the diagnosis with patients, in clinical practice the direction of the discussion is often determined by practical considerations. Patients with moderate to severe dementia usually do not have significant comprehension or retention of the diagnostic information, rendering a review of the diagnosis a futile exercise. Many of these patients do, in fact, have an intuition that something is wrong with them generally, but the specific diagnostic information does not usually add very much to this intuitive sense.

Patients with mild dementia may be able to comprehend and retain diagnostic information, ${ }^{3}$ and it is with this patient population in mind that the debate exists over what and how much to disclose, particularly if there are already clinically significant symptoms of depression, anxiety, or psychosis. Many patients, particularly if lacking insight into their symptoms, may in fact not have very much interest in the outcome of a diagnostic assessment they did not want nor felt they needed. Clinicians often follow the lead of the patient, providing basic diagnostic information to the patient, assessing patient comprehension and desire to learn more, and sharing further information based on the results of that inquiry.

As already noted, the absence of a mutually shared agenda for the physician-patient interaction, such as when the patient does not comprehend or remember the diagnosis, or when diagnostic information is withheld from the patient, can profoundly affect the physicianpatient relationship. This effect on the physician-patient relationship is a consequence of the reality of a lack of shared agenda for the interaction, and not due to any malice or inappropriate behavior on the part of the phy- sician. Thus, the potential effect on the physician-patient relationship can be realized even in instances when the withholding of diagnostic information from the patient may be considered ethically justifiable.

\section{WHOM DOES TREATMENT SERVE?}

Neuropsychiatric symptoms of depression, anxiety, apathy, sleep disturbance, psychosis, and aggression (among others) are common in patients with dementia. ${ }^{8}$ Although these symptoms are often bothersome and even frightening to the patient, in some cases the patient may be unaware of the symptoms, and the primary negative impact then is upon the family or others involved in the care. In such cases, when treatments are recommended, or when treatment outcome is assessed, an important goal is the reduction in caregiver burden. ${ }^{9,10}$ In cases when treatment is burdensome to the patient in terms of side effects, cost, or risk of adverse events, ${ }^{11}$ further questions about the definition of the physician-patient relationship arise. The same is true when similar situations are confronted in the nursing home setting.

In many instances, the interests of the caregivers or care-providers and the interests of the patient overlap considerably. For example, when it is better for the patient to reside at home than in an institutional setting, the caregivers must be sufficiently stable and unburdened emotionally to be able to maintain the provision of care at home, and then treatment of the neuropsychiatric symptoms contributing to the caregiver burden is in the best interests of both patient and family. Similarly, if a particular nursing home is better for a patient but continuation of the current behavior disturbance is incompatible with remaining there, then treatment of the behavioral symptoms clearly serves the interests of all parties and there is no actual conflict of interest.

In other situations, however, the overlapping of interests may be less clear. What if the family or nursing home could manage the patient's disruptive behavior by providing a personal companion for 12 hours per day, but the economic cost of this, though affordable, was seen as undesirable and so served as the basis for a request for more aggressive medical interventions? What if the doctor has a financial stake in the nursing home? What if the neuropsychiatric symptoms cause only a mild caregiver burden or are simply an annoyance to those in the environment? The physician must tread carefully among the apparently competing interests of the defined patient, the care-providers, and his or her own personal interests (should such exist).

\section{FAMILY-CENTERED CARE FOR DEMENTIA}

The model of physician-patient relationship that often best suits the care of dementia patients draws upon ele- 
ments of family-centered care, a model used extensively in pediatrics. ${ }^{12}$ This approach emphasizes the fact that families and other caregivers are often the major source of support in the life of a patient and that their perspective is an important one in medical decision-making. It also emphasizes education and emotional support, including family members and caregivers apart from the identified patient. ${ }^{12}$

In a family-centered care model, the person with the disease is still defined as the primary patient, and his or her welfare and needs are considered primary. Nonetheless, this model creates a natural expectation that family considerations will be explored during decision-making and that needs for family support and education will be addressed by the physician as a routine part of the clinical care. In recognition of the fact that as patient independence diminishes during the natural progression of dementia and the patient becomes more dependent upon, and intertwined with, his or her immediate environment, the object of clinical attention becomes not only the individual patient but the environment as well.

This approach most accurately reflects and accommodates the practical realities of the clinical setting of dementia care. It is mandatory to obtain clinical information from caregivers in order to establish the initial dementia diagnosis, identify neuropsychiatric symptoms, and monitor progress of treatment and progression of the illness. It is likewise mandatory to provide treatment instructions to a caregiver and not only to the patient. Moreover, without proper education of the family, including diagnostic disclosure and explanation of the projected natural history of the illness, it is difficult if not impossible for caregivers to plan for necessary care. Finally, clinical experience as well as a significant body of literature shows that improving the emotional wellbeing of dementia caregivers is not only a humane and compassionate endeavor in itself, but also has significant positive effects on the welfare of the patient. ${ }^{13}$

\section{CONCLUSION}

The medical care of patients with dementia can place unique demands upon, and even alter, the physicianpatient relationship. Certain features of the physicianpatient relationship may be lacking, particularly those that emphasize the mutuality usually inherent in this relationship, such as sharing a common understanding of and commitment to its goals, full disclosure of information, and an emphasis on patient autonomy. The scope of the physician-patient relationship is often broadened to include family members and caregivers, drawing on features of models such as family-centered care. In all cases, the physician is challenged to provide humane and empathic care, even when symptoms of the patient's illness activate psychological forces within the physician that facilitate objectification of the patient.

\section{REFERENCES}

1. Blass DM, Rye RM, Robbins BM, et al. Ethical issues in psychiatric mobile treatment with homebound elderly patients: the PATCH (Psychogeriatric Assessment and Treatment in City Housing) experience. J Am Geriatr Soc 2006;54:843-848.

2. Werner P, Gafni A, Kitai E. Examining physician-patient-caregiver encounters: the case of Alzheimer's disease patients and family physicians in Israel. Aging Ment Health 2004;8:498-504.

3. Karlawish JH, Casarett DJ, James BD, Xie SX, Kim SY. The ability of persons with Alzheimer's disease (AD) to make a decision about taking an AD treatment. Neurology 2005;64: 1514-1519.

4. Bamford C, Lamont S, Eccles M, Robinson L, May C, Bond J. Disclosing a diagnosis of dementia: a systematic review. Int $\mathrm{J}$ Geriatr Psychiatry 2004;19:151-169.

5. Holroyd S, Snustad DG, Chalifoux ZL. Attitudes of older adults on being told the diagnosis of Alzheimer's disease. J Am Geriatr Soc 1996;44:400-403.

6. Maguire CP, Kirby M, Coen R, Coakley D, Lawlor BA, O'Neill D. Family members' attitudes toward telling the patient with Alzheimer's disease their diagnosis. BMJ 1996;313:529-530.

7. Carpenter B, Dave J. Disclosing a dementia diagnosis: a review of opinion and practice, and a proposed research agenda. Gerontologist 2004;44:149-158.

8. Lyketsos CG, Steinberg M, Tschanz JT, Norton MC, Steffens DC, Breitner JC. Mental and behavioral disturbances in dementia: findings from the Cache County Study on Memory and Aging. Am J Psychiatry 2000;157:708-714.

9. Brodaty H, Green A. Defining the role of the caregiver in Alzheimer's disease treatment. Drugs Aging 2002;19:891-898.

10. Lingler JH, Parker LS, DeKosky ST, Schulz R. Caregivers as subjects of clinical drug trials: a review of human subjects protection practices in published studies of Alzheimer's disease pharmacotherapies. IRB 2006;28:11-18.

11. Schneider LS, Dagerman K, Insel PS. Efficacy and adverse effects of atypical antipsychotics for dementia: meta-analysis of randomized, placebo-controlled trials. Am J Geriatr Psychiatry 2006;14: 191-210.

12. Committee on Hospital Care, American Academy of Pediatrics. Family-centered care and the pediatrician's role. Pediatrics 2003; 112:691-697.

13. Mittelman MS, Haley WE, Clay OJ, Roth DL. Improving caregiver well-being delays nursing home placement of patients with Alzheimer disease. Neurology 2006;67:1592-1599. 